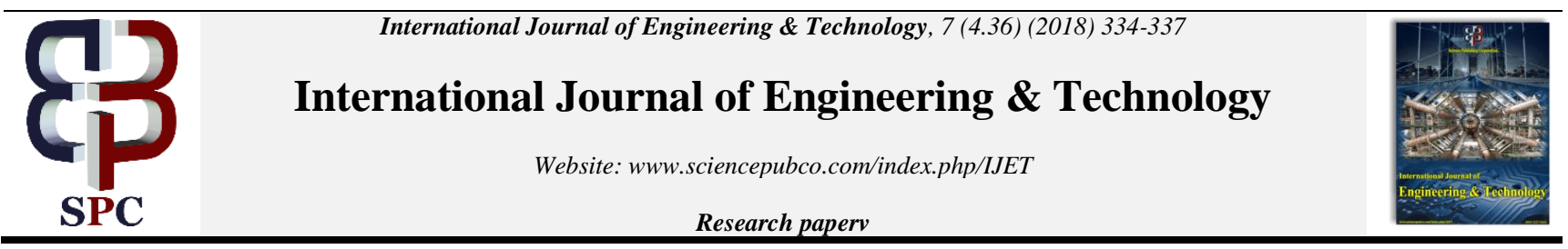

\title{
Analysis of Intensity Distribution in the He-Ne Laser Beam by Gaussian Distribution Function
}

\author{
Ahmed N. Abd ${ }^{1}$, Bahaa Jawad Alwan'1, Wasna'a M. Abdulridha² \\ ${ }^{\text {I}}$ Physics Department, Science Faculty, Al- Mustansiriyah University, Baghdad, Iraq \\ ${ }^{2}$ Basic science Department, College of Dentistry, University of Kufa, Najaf, Iraq \\ *Corresponding author E-mail:wasnaa.albaghdadi@uokufa.edu.iq
}

\begin{abstract}
The purpose of this research is to analyze the distribution of density in the laser beam, both from the experimental and theoretical point of view. I measured the density distribution through the He-Ne laser beam and prepared an attempt to compare it with the Gaussian distribution function. Image profiling can be used to determine the distance of objects depending on the distribution of the laser beam spot surroundings by their width. It can also be used to take pictures of things and define their distances and shape things. As we see, Nubia is very good. From the compatibility analysis found that (the program that made the fitting mention of GNUPLOT).
\end{abstract}

Keyword: Intensity Distribution, Functio, Gaussian

\section{Introduction}

lasers are devices that amplify or increase the intensity of light to produce a beam with high direction and high density and usually have a very pure wavelength or wavelength. Packages come in sizes ranging from ten human hair to a very large building diameter. [1] Since the establishment of the first laser beam in 1960, detection techniques have been developed in order to identify and analyze package properties. In general, laser beam analysis depends on energy measurement, laser beam intensity distribution, beam deflection, waist parameter, number of patterns, etc. [2].In optics, the Gaussian beam is an example of electromagnetic waves, which are well measured by field distributions and transverse power density by Gaussian functions. Many lasers produce gauze-like rays, in which case the laser is said to operate in the primary transverse mode. Goss is commonly used in theoretical and experimental optics, and muscle representation has been successfully applied by many workers, and the mathematical function that describes the shape of the laser beam is the approximate solution of the Helmholtz equation. We obtain this approximation by solving a homogeneous wave equation, and we can derive a wave equation from Maxwell's equations in empty space. Thus, any solution to the Maxwell's equations in empty space meets wavelength equation. [3]

\section{Solution of wave equation oscillating in time and Helmholtz equation}

The waveform can be seen in experiments on diffraction and interference. The light was theoretically depicted as an electromagnetic wave that verified Maxwell's equations. In contrast, the nature of light molecules is expressed by the idea of re-measurement or photon in the theoretical description of electromagnetic field estimation. However, the degree of coherence of laser light is much better than other forms of light, and in exceptional cases only the intensification of the electromagnetic field of laser light is reflected in any intrinsic effect. Therefore, we will explain in detail the propagation of light, especially the ultra-directional light (and most of the microscope) of the laser, starting with Maxwell's equations. [4] From electromagnetic theory, E,H are the electric field and magnetic field respectively, magnetic flow density $\vec{B}$, electrostatic flow density $\vec{D}$, power density $\vec{J}$, and charge density $\rho$, all may change as coordinates functions (x, y, z) and time t, Are linked to

Maxwell's equations: $\nabla \times \vec{E}=-\frac{\partial \vec{B}}{\partial t}$,

$\nabla \times \vec{H}=\vec{J}+\frac{\partial \vec{D}}{\partial t}$

$\nabla \cdot \vec{D}=\rho$,

$\nabla \cdot \vec{B}=0$

Here $\nabla$ is the vector operator with $\partial / \partial x, \partial / \partial \mathrm{y}$ and $\partial / \partial Z$ as its $x, y$, and $\mathrm{Z}$ components, respectively. Let $\varepsilon$ denote the electric permittivity, $\mu$ the magnetic permeability and $\sigma$ the electric conductivity of medium. We have then

$\vec{D}=\varepsilon E, \vec{B}=\mu \vec{H}, \vec{J}=\sigma \vec{E}$.

By using the polarization $\vec{P}$ and the permittivity in vacuo $\varepsilon 0$, we have

$\vec{D}=\varepsilon_{0} \vec{E}+\vec{P}$

The electric susceptibility is given by: 
$\vec{P}=\varepsilon_{0} x \vec{E}$

In general, $\vec{P}$ it is proportional to the time when the electric field is weak, but is no longer proportional with $\mathrm{E}$ when it is strong. In addition, this does not always follow a time difference according to the time change

The $\vec{P}$-field follows the $\vec{E}$-field only if $x$ is constant, i.e. independent on the frequency of external field. We shall assume later that the medium is dielectric so that $\sigma=0$, and the permeability is :

$\mu=\mu 0=4 \pi \times 10^{-7} \mathrm{H} / \mathrm{m}$.

Applying the curl operation to both sides of (1) and using (2) and $\vec{B}=\mu \vec{H}$, we obtain:

$\nabla_{\mathrm{X}}(\nabla \times \vec{E})=-\nabla \times \frac{\partial \overrightarrow{\mathrm{B}}}{\partial \mathrm{t}}=-\mu \frac{\partial}{\partial t} \nabla \times \vec{H}=-\mu \frac{\partial^{2} \vec{D}}{\partial^{2} t}$.

According to vector calculus we have

$\nabla(\nabla \times \vec{E})=\nabla(\nabla \cdot \vec{E})-\nabla^{2} \vec{E}$,

so that (8) can be written as

$\nabla(\nabla \cdot \vec{E})-\nabla^{2} \vec{E}=-\mu \frac{\partial^{2} \vec{D}}{\partial^{2} t}$

Using (3.4) and $\vec{D}=\varepsilon \vec{E}$ we have

$\nabla . \vec{E}=\frac{1}{\varepsilon} \nabla \cdot \vec{D}=\frac{\rho}{\varepsilon}$

An electric charge produces only an electrostatic field and is irrelevant to electromagnetic waves in an optical medium, we can neglect it and put $\rho=0$. Therefore, we have $\nabla \cdot \vec{E}=0$ and equation (10) becomes:

$\nabla^{2} \vec{E}-\varepsilon \mu \frac{\partial \vec{E}}{\partial t}=0$

This is the equation of waves propagating with the velocity $\mathrm{v}$ such that $\mathrm{v}^{2}=\frac{1}{\varepsilon \mu}$, and the velocity of light in vacuo is $\mathrm{c}=\frac{1}{\sqrt{\varepsilon 0 \mu 0}}$.

Using Fourier expansion one can express any waveform as superposition of harmonic waves . On the other hand . laser light is almost perfectly monochromatic. Therefore, we can express the time factor of monochromatic electromagnetic wave of frequency $\omega$ by $\vec{E}=\vec{\varepsilon} \exp (i w t)$, and the wave equation (12) becomes:

$\nabla^{2} \vec{\varepsilon}+k^{2} \vec{\varepsilon}=0$

where $\mathrm{k}^{2}=\omega^{2} \varepsilon \mu$, and $\mathrm{k}=\frac{\omega}{v}$ is the wave number.

In normal waveform processing, it is sufficient to use the waveform of the scalar $\mathrm{u}$ variable:

$\nabla^{2} u+k^{2} u=0$.

This equation is known as the Helmholtz equation. In order to explain diffraction, interference, degeneration, etc., this is equivalent to talking only about a vector component. In general, when the dimensions of the medium are large compared to the wavelength, the optical wave is purely cross-sectional so that the numerical calibration is the plant. We will use the Helmholtz equation to analyze the properties of monochromatic light beam, and it is known that any electromagnetic field can be arbitrarily extended to flat or spherical waves, but the light beam that spreads along the axis can extend roughly to the Hermian Gaussian roads along this axis [5 ].Taking the $\mathrm{z}$ axis along the light beam and the wave number of the medium for transverse waves as $\mathrm{k}$, we put

$u=A(x, y, z) \exp (-i k z)$

The function $A$ representing the light beam must become practically zero for large values of $x$ or $y$ and changes slowly with z. Substituting equation (3.15) in (3.14) we get:

$\left(\frac{\partial^{2} A}{\partial X^{2}}\right) \exp (-i k z)+\left(\frac{\partial^{2} A}{\partial y^{2}}\right) \exp (-i k z)+\left(\frac{\partial^{2} A}{\partial z^{2}}\right) \exp (-i k z)$

$-2 i k\left(\frac{\partial A}{\partial z}\right) \exp (-i k z)-k^{2} \mathrm{~A} \operatorname{erp}(-i k z)+k^{2} A \operatorname{erp}(-i k z)=0$,

which reduces to

$\left(\frac{\partial^{2} A}{\partial x^{2}}\right)+\left(\frac{\partial^{2} A}{\partial y^{2}}\right)-2 i k\left(\frac{\partial A}{\partial z}\right)+\left(\frac{\partial^{2} A}{\partial z^{2}}\right)=0$

The term $\exp ^{-}(i k z)$ of Equation 15 accounts for the wave os cillation along the propagation direction. The dependence of $A$ on 2 is of a different nature. It likely accounts for the slow decrease in the amplitude of the wave as the wave propagates. Thus we can say that $A$ varies slowly with $\mathrm{z}$, and thus we can neglect the term $\left(\frac{\partial^{2} A}{\partial Z^{2}}\right)$ in front of the other ones and drop it from Equation (17) . The resulting equation is

$\left(\frac{\partial^{2} A}{\partial x^{2}}\right)+\left(\frac{\partial^{2} A}{\partial y^{2}}\right)-2 i k\left(\frac{\partial A}{\partial z}\right)=0$

Equation (18) is called the paraxial wave equation.

\section{Approximate solution of the Helmholtz equa- tion}

The simple solution of the wave equation is the solution in which we enter the simplest forms of solution and find the exact form that obeys the equation of the wave. The most formal solution is the solution where we solve the wave equation in full. We believe that the simple experimental solution for (18) of the model

$A\left(\overrightarrow{r)}=F_{1}(z) \exp \left[-\frac{\rho^{2}}{F 2(Z)}\right]\right.$.

Here $F_{1}(z)$ and $F_{2}(z)$ are slowly varying functions of $z$ only, and $\rho^{2}=x^{2}+y^{2}$. To find the equations which describe $F_{1}(z)$ and $F_{2}(z)$ we will substitute the equation (19) into the equation 18). The first derivative of function $A$ with respect to $x$ is given by

$\frac{\partial A}{\partial x}=F_{1}(z)\left[-\frac{2 x}{F_{2}(z)}\right] \exp \left[-\frac{\rho^{2}}{F_{2}(z)}\right]$,

and the second derivative

$\frac{\partial^{2} A}{\partial x^{2}}=F_{1}(Z)\left[-\frac{2}{F_{2}(z)}\right] \exp \left[-\frac{\rho^{2}}{F_{2}(z)}\right]+F_{1}(z)\left(-\frac{2 x}{F_{2}(z)}\right)^{2} \exp \left[-\frac{\rho^{2}}{F 2(z)}\right]$

and similarly for the derivative with respect to $y$

$\frac{\partial^{2} A}{\partial y^{2}}=F_{1}(Z)\left[-\frac{2}{F_{2}(z)}\right] \exp \left[-\frac{\rho^{2}}{F_{2}(z)}\right]+F_{1}(z)\left(-\frac{2 y}{F_{2}(z)}\right)^{2} \exp \left[-\frac{\rho^{2}}{F 2(z)}\right]$

According to equation (18) we will also find the first derivative of with respect to $\mathrm{z}$ 


$$
\begin{aligned}
& \frac{\partial \mathrm{A}}{\partial \mathrm{z}}=F_{1}{ }_{1}(z) \exp \left[-\frac{\rho^{2}}{F_{2}(z)}\right]+ \\
& \exp \left[-\frac{\rho^{2}}{\mathrm{~F}_{2}(\mathrm{z})} \ldots \ldots \ldots \ldots \ldots \ldots \ldots \ldots \ldots \ldots \ldots \ldots \ldots \ldots \ldots \ldots \ldots\right. \\
&
\end{aligned}
$$

Substituting equations (21), (22), (23) into equation 3.18) we get $-\frac{4 F_{1}(z)}{F_{2}(z)}+F_{1}(z) \frac{4}{F_{2}^{2}(z)}\left(x^{2}+y^{2}\right)-2 i k F_{1}^{\prime}(z)-2 i k \frac{F_{1}(z)}{F_{2}^{2}(z)} F_{2}^{\prime}\left(x^{2}+\right.$ $\left.y^{2}\right)=0, .$.

or

$\left[-\frac{4 F_{1}(z)}{F_{2}(z)}-2 i k F_{1}^{\prime}(z)+\left(x^{2}+y^{2}\right)\left[-\frac{4 F_{1}(z)}{F_{2}^{2}(z)}-2 i k \frac{F_{1}(z)}{F_{2}^{2}(z)} F_{2}^{\prime}(z)\right]=0\right.$ (25)

Equation (25) will be fulfilled if

$\frac{2 F_{1}(z)}{F_{2}(z)}+i k F_{1}^{\prime}(z)=0$

And

$\left.\frac{2 F_{1}(z)}{F_{2}^{2}(z)}-i k \frac{F_{1}(z)}{F_{2}^{2}(z)} F_{2}^{\prime}(z)\right]=0$

separately. It follows from (27) that

$F_{2}^{\prime}(z)=\frac{2}{i k}$

By integration of the equation (28) we get:

$$
F_{2}(z)=\frac{2 z}{i k}+c
$$

where $\mathrm{c}$ is constant of integration.

We can rewrite the equation (26) as:

$i k F_{1}^{\prime}(z)=-\frac{2 F_{1}(z)}{F_{2}(z)}$

Or

$\frac{F_{1}^{\prime}(z)}{F_{1}(z)}=-\frac{2}{i k} \frac{1}{F_{2}(z)}$

By using the equation (29) in $\mathrm{t} h$ e equation (31) we obtain

$\frac{F_{1}^{\prime}(z)}{F_{1}(z)}=-\frac{2}{i k \frac{2 k}{i k}+c}$.

The last equation can be writ ton as

$\frac{d}{d z} \ln F_{1}(z)=-\frac{1}{Z+\frac{i k}{2} C}$,

Then

$\ln F_{1}(z)=-\ln \left(z+\frac{i k}{2} \mathrm{c}\right)+c_{1}, \ldots \ldots \ldots$

where $c_{1}$ can be written as $c_{1}=\ln B_{1}$.

Then we can write equation (34) in the form

$F_{1}(Z)=\frac{B_{1}}{z+\frac{i k c}{2}}$.

\section{Modeling of the Gaussian laser beam by Ap- proximate solution of Helmholtz equation}

For the purpose of modeling of the Gaussian laser beam via approximate solution of Helmholtz equation we substitute the forms of $F_{1}(\mathrm{z})$ and $F_{2}(z)$, equations (35) and (29) respectively, into equation (19) and we get

$\mathrm{A}(\vec{r})=\frac{B_{1}}{Z+\frac{\mathrm{ikc}}{2}} \exp \left[-\frac{\rho^{2}}{\frac{z_{2}}{i k}+c}\right]$,

This equation represents the spread of the Gaussian beam in the direction of $\mathrm{z}$. For each $\mathrm{z}$ value, the intensity is a gausic function of the half-way distance $\rho$. This is why the wave is named by a Gosi package. The Gauss function peaks at $\rho=0$ (on the axis) and decreases monotonously with an increase of $\rho$. The beam radius $w$ (z) is increased for Gaussian distribution with the axial distance. The large beam deviation corresponds to the radius of the beam beam with poor beam quality. Low beam deflection can be important for applications such as optical signal communication or free space. [6]

\section{Measurement of the intensity distribution in He-Ne laser}

The theoretical package (TEM00) has a perfect guise. The laser can produce many other TEM modes. In general, one could say that laser beams have a symmetric density profile. For example: if we pass through the beam, the minimum intensity is on the edge and as we move towards the center, it increases and reaches the maximum in the center and then falls in a similar way to the other end. For the current measurement, we used a He-Ne laser beam $(\lambda$ $=562.8 \mathrm{~nm}$ ) with 5 megawatt power and a high-speed silicon detector to measure the intensity of the laser light falling on the detector at the laser beam that does not weaken. The detector is located 0.4 meters from the end of the laser output and moved to a transient phase in mm increments, as shown in Fig. 1.

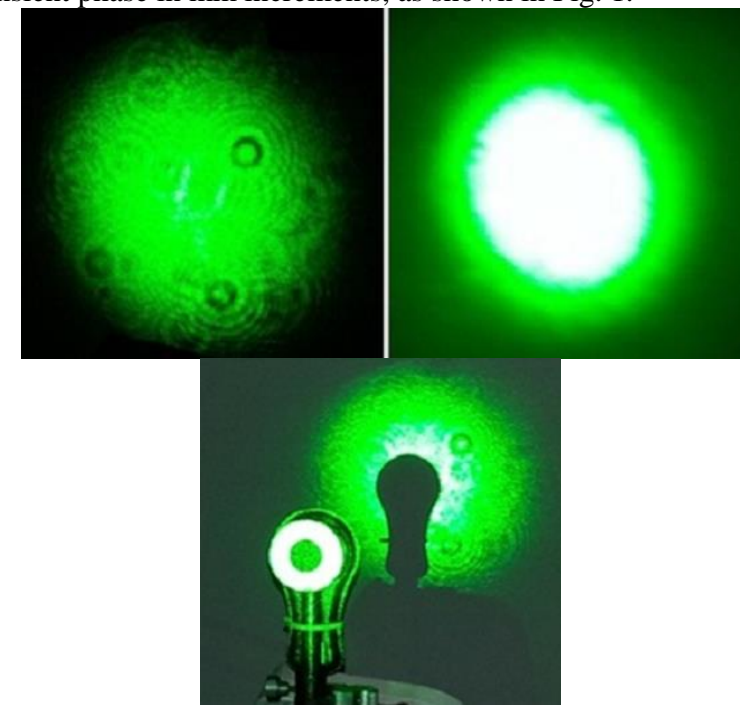

Figure 1: Scanning detector along diameter beam.

The data were plotted and compared to the theoretically predicted Gaussian model. The theoretical gouse distribution contains three coefficients: I0 maximum intensity, $\mathrm{x} 0$-centre of beam (maximum intensity point), $\mathrm{w}$ width or beam radius ( $1 / 2$ diameter). The Gaussian curve was suitable for measuring data using GNUPLOT. For each measured point, the difference between the theoretical fit and the measured value was saved in an "error" cell, and the sum of these values was shown to show compatibility quality". I was able to adjust the theoretical graph constants so that the quality of the fit was almost perfect (minimum value is best suited). 


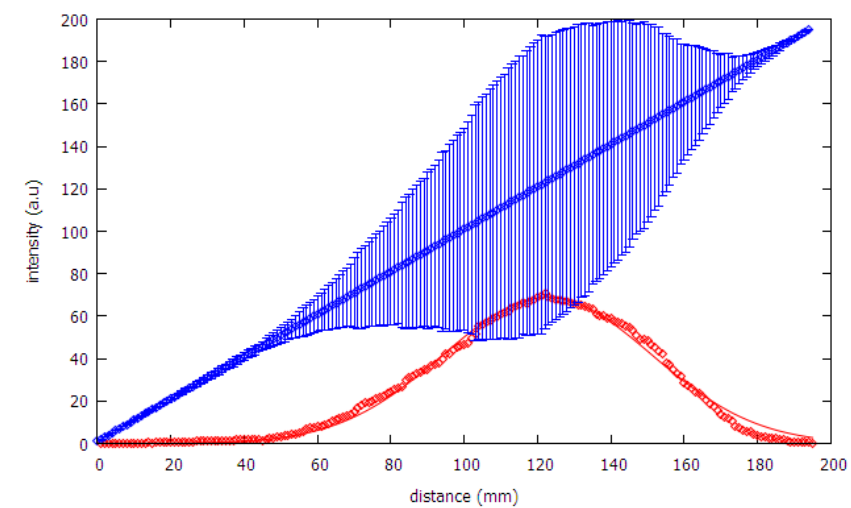

Figure 2: Results of measurement and fit to the Gaussian curve with distance $\mathrm{x}$-axes

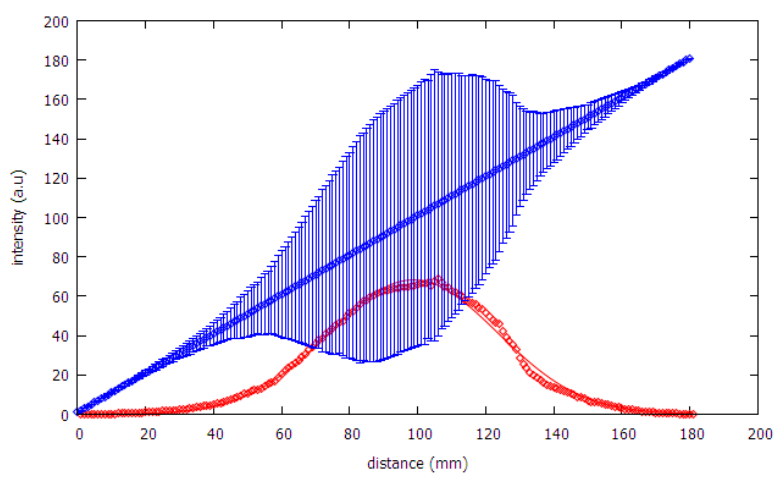

Figure 3: Results of measurement and fit to the Gaussian curve with distance $y$-axes.

Final set of parameters

$\mathrm{x} 0=104: 484$

$\mathrm{w}=70: 3211$

I $0=147: 568$

Asymptotic Standard Error

$+/-0.3436(0.3289 \%)$

$+/-0.6883(0.9788 \%)$

+/- $1.249(0.8463 \%)$.

\section{Conclusions}

- $\quad$ The mathematical equation that describes the Gaussian beam can be obtained from the approximate solution of the Helmholtz equation that follows the wave equation.

- $\quad$ In this paper we presented a precise solution to the Gaussian wave wave. Our solution satisfies Maxwell's equations. This exact solution was compared with the results obtained previously by other workers, particularly the axial holographic scale and axial axial coordinates values.

- $\quad$ The theme of the gothic symptoms provides students with basic understanding of the physics and diffusion of the laser beam. Due to the widespread use of laser today, this material must be an essential part of the course on optics. Coverage of high-level Gaussian patterns further deepens the discussion of light waves and confirms the main components of wave function: amplitude and phase.

- The quality of the Gaussian beam (control of top hat symmetry and laser beam capacity assessment) is very important in medical applications, especially in surgical operations. The intensity distribution of the laser beam is strongly related to the strength of the source and can be used to determine the distance of objects.

\section{References}

[1] Elijah Kannatey-Asibu, Jr., IPrinciples of Laser Materials Processing," John Wiley \& Sons, Inc.2009.

[2] Heard, H.G., 1968. Laser Parameter Measurements Handbook. John Wiley and sons Inc.

[3] Fleischer, J.M. and D. Doggett, 1989. Spectral Profiling with a single photodiode. Laser and Optoelectronics , pp: 47-52.
[4] Hull, D.M . And A. Stewart, 1985. Laser beam profiles principles and definitions. Laser and Application, pp: 75.

[5] K. Shimoda, , "Introduction to Laser Physics", Second Edition, Springer, 1986

[6] Javier Alda, Laser and Gaussian Beam Propagation and Transformation," Optics Department. University Complutense of Madrid.School of Optics 2001 (article). 